Respiration 2010;80:260-261

DOI: $\underline{10.1159 / 000314981}$

\section{Serosal Involvement in Adult-Onset Autoinflammatory Disorders}

\author{
Luca Cantarini ${ }^{\mathrm{a}}$, Orso Maria Lucherini ${ }^{\mathrm{b}}$, Rolando Cimaz ${ }^{\mathrm{c}}$, \\ Maria Giuseppina Brizi ${ }^{\text {a }}$, Mauro Galeazzi ${ }^{\text {a }}$ \\ a Interdepartmental Research Center of Systemic Autoimmune \\ and Autoinflammatory Diseases, Policlinico Le Scotte, and \\ ${ }^{b}$ Department of Evolutionary Biology, University of Siena, \\ Siena, and ' Department of Pediatrics, Rheumatology Unit, \\ Anna Meyer Children's Hospital and University of Florence, \\ Florence, Italy
}

We read with great interest the article by Lega et al. [1], who described a patient with familial Mediterranean fever (FMF) who had febrile episodes of right-sided pleuritis, in absence of any extrathoracic complaints. The patient did not fulfill the diagnostic criteria for FMF [2], nevertheless colchicine administration led to the complete resolution of attacks, and genetic testing further strengthened the presumptive diagnosis, since the patient showed a homozygous M694I/M694I mutation in the MEFV gene. The article by Lega et al. suggests that autoinflammatory disorders, mainly when the onset is in adulthood, may sometimes present with isolated serosal involvement. Since an increasing interest is currently being devoted to serositis and autoinflammatory disorders [3], we would like to present some additional data on our personal experience with adult-onset autoinflammatory disorders and their presentation with isolated serosal involvement occurring with fever.

To date, among monogenic periodic fever syndromes, a late onset of symptoms is reported both for FMF [4], up to the age of 65 [5], and for tumor necrosis factor receptor-associated periodic syndrome (TRAPS) [6-8]. FMF, caused by mutations in the $M E F V$ gene, and TRAPS, caused by mutations in the TNFRSF1A gene, are respectively the most common autoinflammatory recessive and autosomal dominant disorders and are characterized by recurrent episodes of fever associated with abdominal and chest pain, arthralgia and/or arthritis, and cutaneous manifestations. Adult-onset FMF is usually related to low-penetrance mutations, and patients may experience milder disease, although the clinical manifestations are usually similar to those of younger patients, with the exception of arthritis and erysipelas-like erythema, which are significantly less frequent in adults [3]. Disease onset in adulthood has been described for TRAPS as well, up to the age of
63 [6], and as with FMF, is frequently related to low-penetrance mutations [6-8]. Patients with adult-onset TRAPS may be characterized by a phenotype that can mimic FMF in the duration of inflammatory attacks, which may last $<1$ week [9]. In addition, patients with adult-onset TRAPS may present with atypical clinical manifestations, such as recurrent serosal involvement of pericardium as the sole clinical manifestation [6-8]. Among these subjects, we recently hypothesized the criteria (positive family history and poor response to colchicine) for identifying patients for whom testing for genetic mutations of the TNFRSF1A gene should be carried out $[7,8]$. In addition, with the aim of improving the genetic diagnosis in adults with suspected autoinflammatory disorders, we have recently identified some variables that appear to be strongly related to the probability of detecting gene mutations in MEFV and TNFRSF1A and we have also developed a diagnostic score for identifying patients at high risk of carrying these mutations (unpublished data, paper submitted). Our score, since the thoracic involvement (pleural and/or pericardial) was included, in addition to the clinical criteria that we previously suggested [7, 8], might also represent an evidence-based guideline, thus assisting in the diagnostic evaluation of patients with recurrent fever and isolated serosal involvement, and could help identify the few patients among them who may prove to be carriers of mutations in 'autoinflammatory genes'.

In conclusion, we strongly believe that adults presenting with idiopathic recurrent serositis should be evaluated for mutations in the genes responsible for FMF and TRAPS in order to identify patients with FMF and TRAPS, since through lack of an appropriate treatment, they may be at high risk of developing secondary renal amyloidosis. However, genetic testing of all patients presenting with a recurrent inflammatory serosal involvement might have low efficiency and elevated costs. Therefore, more research is needed in order to identify evidence-based criteria for selecting the patients who need to be genetically analyzed for mutations in the autoinflammatory genes.

\section{References}

1 Lega JC, Khouatra C, Cottin V, Cordier JF: Isolated recurrent pleuritis revealing familial mediterranean fever in adulthood. Respiration 2010;79:508-510.

$\checkmark 2$ Livneh A, Langevitz P, Zemer D, Zaks N, Kees S, Lidar T, et al: Criteria for the diagnosis of familial Mediterranean fever. Arthritis Rheum 1997;40:1879-1885.

-3 Cantarini L, Imazio M, Brucato A, Lucherini OM, Galeazzi M: Innate versus acquired immune response in the pathogenesis of recurrent idiopathic pericarditis. Autoimmun Rev 2010;9:436-440.

\section{KARGER}

Fax +41613061234 E-Mail karger@karger.ch www.karger.com
(C) 2010 S. Karger AG, Base

0025-7931/10/0803-0260\$26.00/0 
4 Sayarlioglu M, Cefle A, Inanc M, Kamali S, Dalkilic E, Gul A, et al: Characteristics of patients with adult-onset familial Mediterranean fever in Turkey: analysis of 401 cases. Int J Clin Pract 2005;59:202-205.

5 Cantarini L, Capecchi PL, Lucherini OM, Laghi Pasini F, Galeazzi M: Familial Mediterranean fever diagnosed in an elderly patient. Clin Exp Rheumatol, in press.

6 Dodé C, André M, Bienvenu T, Hausfater P, Pêcheux C, Bienvenu J, et al; French Heraditary Recurrent Inflammatory Disorder Study Group: The enlarging clinical, genetic, and population spectrum of tumor necrosis factor receptor-associated periodic syndrome. Arthritis Rheum 2002;46:2181-2188.

$\checkmark 7$ Cantarini L, Lucherini OM, Cimaz R, Baldari CT, Bellisai F, Rossi Paccani $\mathrm{S}$, et al: Idiopathic recurrent pericarditis refractory to colchicine treatment can reveal tumor-necrosis factor associated periodic syndrome. Int J Immunopathol Pharmacol 2009;22:1051-1058.
8 Cantarini L, Lucherini OM, Baldari CT, Laghi Pasini F, Galeazzi M: Familial clustering of recurrent pericarditis may disclose tumor necrosis factor receptor-associated periodic syndrome. Clin Exp Rheumatol, in press.

9 Dodé C, Papo T, Fieschi C, Pêcheux C, Dion E, Picard F, et al: A novel missense mutation (C30S) in the gene encoding tumor necrosis factor receptor 1 linked to autosomal-dominant recurrent fever with localized myositis in a French family. Arthritis Rheum 2000;43:1535-1542.

Luca Cantarini

Unit of Rheumatology, Policlinico Le Scotte

University of Siena, Viale Bracci 1

IT-53100 Siena (Italy)

Tel. +390347 938 5457, Fax +390577 40450

E-Mail cantariniluca@hotmail.com 